\title{
A simultaneous multiple BeiDou signal acquisition algorithm for a software- based GNSS receiver
}

\author{
Fei Xie ${ }^{a, *}$, Jianye Liu ${ }^{a}$, Rongbing Li ${ }^{a}$, Shaojun Feng ${ }^{b}$ \\ a Navigation Research Center, Nanjing University of Aeronautics and Astronautics, Nanjing 210016, \\ China ${ }^{b}$ Department of Civil and Environmental Engineering, Imperial College London, London, UK
}

\begin{abstract}
The time needed for a Global Navigation Satellite System (GNSS) receiver to acquire satellite signals is one of the key parameters to assess the performance of a receiver. This presents a challenge for a software receiver in which more frequency bins and satellite codes are required to search using conventional meth-ods. In order to speed up the acquisition process of the BeiDou software receiver in a cold start, this paper proposes a simultaneous multiple Code Division Multiple Access (CDMA) signal acquisition algorithm. The time for searching satellites is reduced by constructing new local codes to enable the capability of detecting the presence of multiple BeiDou satellites. Besides, the individual phase identification approach for each visible satellite is further presented with multiple satellites acquisition. So a search within the PRNs used for the generation of local combined code is performed. Finally, the test results show that the proposed acquisition algorithm can effectively detect multiple BeiDou satellites simultaneously. In addition, the test results show that signal acquisition time can be reduced by $8.7 \%$ to $24.1 \%$ depending on different combination numbers and different numbers of visible satellites in the combination list.
\end{abstract}

\section{Introduction}

Global Positioning System (GPS) is one of the most successfully technique achievements. It can be found a lot of applications in many areas such as transport, mobile network synchronization and social networking. However, GPS signal is even weaker than thermal noise when it reaches the Earth surface. The other similar navigation systems including GLONASS, regional operational BeiDou and future operational Galileo have brought both opportunities and challenges for navigation system design, including GNSS/INS integrated system. The BeiDou navigation satellite system is independently built and operated by China, which will consist of 30 non-geostationary and 5 geostationary satellites (GEO). According to its overall planning schedule, the system is planned to be established completely and provide global service by 2020. Moreover, it is compatible with other global satellite navigation systems. As the 16th BeiDou satellite was launched on October 25,2012 , the system has provided regional Asia Pacific service since early year 2013, with a constellation of five satellites in geostationary orbit, five in inclined geostationary orbit (IGSO) and four in

medium earth orbit (MEO). In the near future, BeiDou navigation satellite system will independently provide open services for global users, and particularly provide high quality services in Asia Pacific region.
BeiDou software receiver provides full access to base band signals processing based on intermediate frequency (IF) signal inside the receiver channels. The first critical stage is the acquisition block that aims to determine visible satellites and phase/frequency of the received signals. The acquisition stage usually needs to perform a correlation operation between the input samples and locally generated replicas of the signals transmitted by the different satellites. In a hardware receiver, the correlation of acquisition algorithm is usually completed in an application-specific integrated circuit (ASIC), and the serial search acquisition (SSA) is an often-used method which scans all the bins in the code phase and carrier frequency [1]. However, all possible values of the code phase and frequency scanning showed that the serial search acquisition method is a very time-consuming procedure in a software receiver. In order to reduce the computational load, the frequency-domain acquisition methods usually exploit a Discrete Fourier Transform (DFT) or a Fast Fourier Transform (FFT) conversion to implement the correlation operation in the frequency domain [2-4].

To improve the sensitivity of acquisition results, several different techniques, such as non-coherent, coherent and the differentially coherent have been proposed for the acquisition of CDMA signals [5-11]. Besides, expressions for the false alarm and detec-tion probabilities of acquisition have been analyzed $[12,13]$. In 
particular, coherent combing is the optimal processing strategy that provides the highest acquisition sensitivity at the expenses of an increased computational load since non-coherent combing suffers from squaring loss compared to coherent combing [14]. However, the effect of navigation data bit transitions has to be considered when choosing the length of coherent integration time. To eliminate the impact of Doppler frequency, the GPS signal acquisition aided by SINS was proposed for the acquisition of weak signals [15]. However, these researches mainly focus on one satellite or treat multiple satellites acquisition individually.

In order to reduce the search time of signals acquisition in cold start period of receiver, a fast acquisition algorithm capable of acquiring multiple BeiDou satellites' signals simultaneously has been proposed. The fast acquisition is based on parallel phase acquisition approach which can search all the code phase bins simultaneously. Consequently, the search space is reduced from three domains to two domains (PRN and Doppler frequency). The proposed simultaneous multiple satellites acquisition algorithm is achieved by the construction of new local codes which contain combinations of PRNs from different satellites. The new local codes are therefore used to perform correlation processing with the received signals. Upon the detection of the existence of PRNs, a further correlation with one of the code in each combination is carried out in order to identify the signal to be tracked. If the correlation of a new local code resulting no-existence of signals, all the satellite PRNs in this combination can be excluded at the same time. Therefore, the total searching time for non-visible satellites is reduced compared to the traditional parallel acquisition approach.

The rest of this paper is organized as follows. In Section 2 we briefly analyze the QPSK modulation for BeiDou B1 signal in order to lay the foundation for the generation of combined local codes, and then the simultaneous acquisition algorithm for multiple BeiDou satellites is proposed. In Section 3 the phase and frequency identification method for individual visible satellite is described. The computation complexities of different complexity algorithms are also analyzed theoretically. Section 4 shows the test results to demonstrate the capability and performance of the proposed algorithm before the paper is concluded in Section 5 .

\section{A simultaneous multiple BeiDou signal acquisition algorithm}

\subsection{Modulation analysis for BEIDOU signals}

For different GNSS, a number of satellites transmit navigation signals in different modulations on a variety of different frequency bands. According to the BeiDou ICD for open service signal B1 and B2, the BeiDou satellites exploit the Quadrature Phase Shift Keying (QPSK) modulation to transit navigation signals on B1 and B2 frequency bands [16]. Unlike BPSK, QPSK sends two bits of digital information at a time, so the data transmitting rate with QPSK is doubled of that with BPSK. Only B1 signal is considered and studied in this paper. The QPSK modulated waveform is shown in Fig. 1.

The BeiDou B1 signal is the sum of component I (open) and Q (authorization) which are in phase quadrature of each other. The ranging code and navigation message are modulated on carrier. The modulation processing of BeiDou B1 signal can be seen in Fig. 2.

For BeiDou B1 navigation message that has a rate $50 \mathrm{bps}$, a secondary code of Neumann Hoffman $(\mathrm{NH})$ code is modulated on ranging code. As shown in Fig. 3, the period of $\mathrm{NH}$ code is selected as long as the duration of a navigation message bit. According to the BeiDou ICD document, the B1 signal adopts the NH code with length of 20 bits, which the bit duration is $1 \mathrm{~ms}$. It is modulated

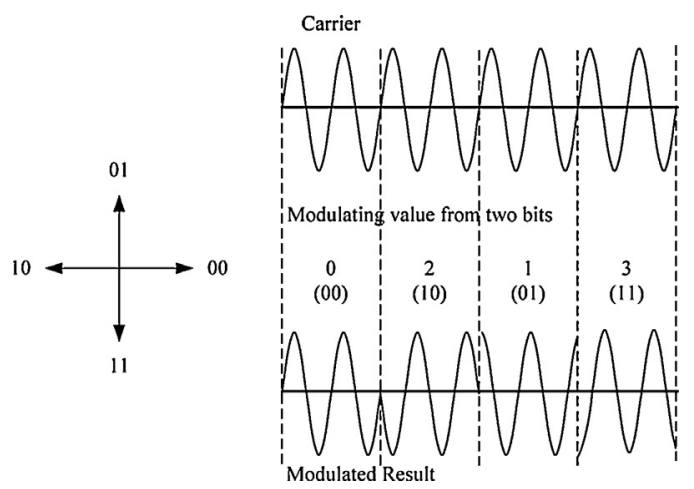

Fig. 1. Modulation waveform of QPSK.

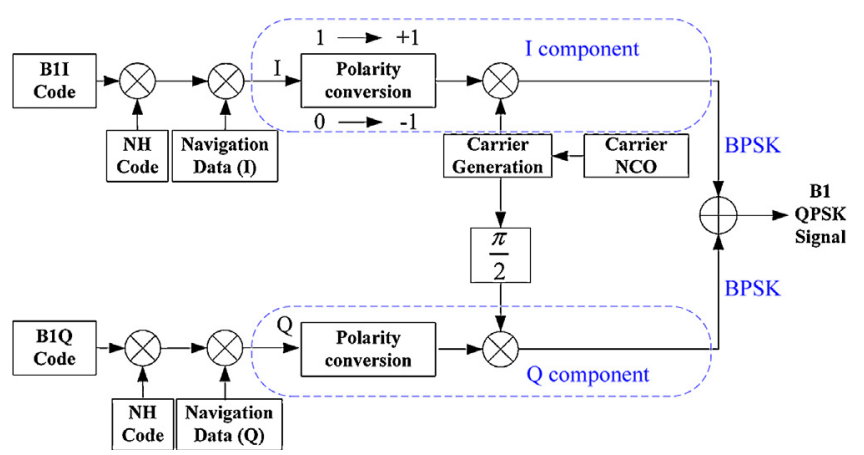

Fig. 2. Modulation processing of BeiDou B1 signal.

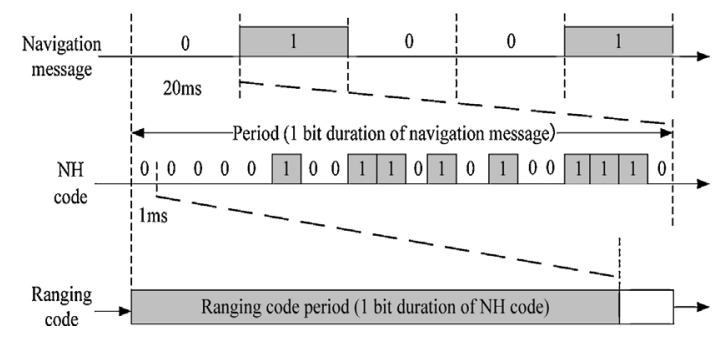

Fig. 3. The NH code period and structure.

on the ranging code synchronously with navigation data. In addition, the Neumann Hoffman (NH) code of BeiDou B1 signal can be removed in period of bit/frame synchronization.

According to the QPSK modulation principle, two navigation data bits are transmitted simultaneously with the BPSK modulation. One data bits produce the In-phase (I) component and the other data bits produce the Quadrature (Q) component. So the mathematical expression of a BeiDou B1 IF signal can be represented by:

$$
\begin{aligned}
S_{k}^{j}= & A_{C} D_{I}^{j}(t) C_{I}^{j}\left(t-\tau_{I}\right) \cos \left[2 \pi f_{c} t+\phi_{I}^{j}\right] \\
& +A_{C} D_{Q}^{j}(t) C_{Q}^{j}\left(t-\tau_{Q}\right) \sin \left[2 \pi f_{c} t+\phi_{Q}^{j}\right]+n_{C j}=S_{I}^{j}+S_{Q}^{j}+n_{C j}
\end{aligned}
$$

where $A_{c}$ is amplitude of BeiDou B1 IF signal; $D_{1}(t)$ and $D_{1}(t)$ are the navigation data respectively modulated with the $\mathrm{NH}$ code in component I and Q; $\tau_{I}$ and $\tau_{Q}$ denotes the code chips corresponding with time delay of components I and Q respectively; $f_{c}$ is the carrier frequency of BeiDou IF signals; $\phi_{I}, \phi_{Q}$ are initial phase of IF carrier in components I and $\mathrm{Q} ; n_{C j}$ is received BeiDou IF signal noise.

Comparing with the GPS C/A code has a chipping rate of $1.024 \mathrm{Mbps}$ and a length of 1023 chips, the BeiDou B1 ranging code has a chipping rate of $2.046 \mathrm{Mbps}$, and the length is 2046 chips. Since QPSK modulation consists of two BPSK modulations 


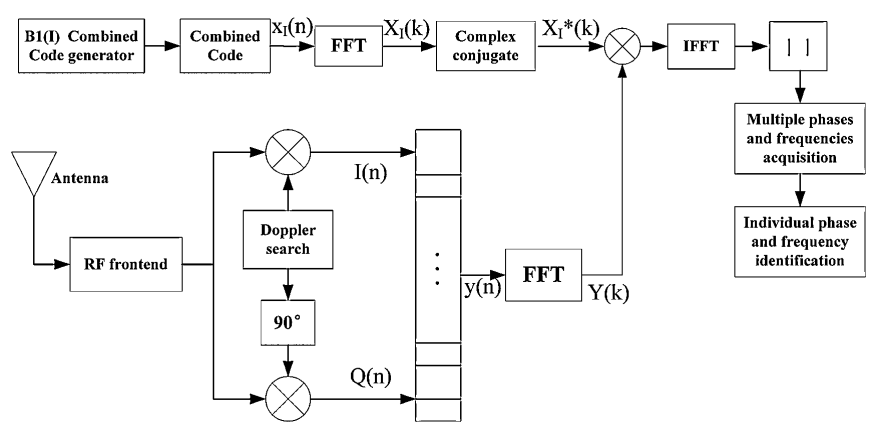

Fig. 4. The block diagram of the proposed acquisition scheme.

on In-phase (I) and Quadrature (Q) components of the signal, the In-phase component and the Quadrature component can be processed respectively as two BPSK signal added. Additionally, only the signal acquisition of I component is considered due to the code demodulation of $\mathrm{Q}$ component is required an authorization.

\subsection{Simultaneous acquisition of multiple BeiDou satellites}

Signal acquisition is the initial process in a BeiDou receiver followed by tracking and extraction of navigation bits. Signal acquisition processing involves search in three domains: code delay, Doppler frequency and satellite. The search in the phase should cover the possible chips of code delay. The search in the frequency should cover the full range of possible Doppler shift. The satellite PRN search should cover all satellites in the BeiDou constellation. The combination of these three types of research requires relative long time. In a multiple channels hardware receiver, more channels are allocated more PRNs in order to improve the search efficiency. In a software receiver, a similar scheme may be used if the frequency or phase parallel processing techniques are employed.

In any case, the total search space is the product of the total numbers of Doppler or code delay bins and total numbers of designed satellite PRNs. The time needed for the acquisition of multiple satellites' signals is even longer. Even though a receiver can shorten the re-acquisition time after lose signal tracking based on the knowledge of previous tracked satellites and navigation data, the re-acquisition efficiency remains a critical issue for a cold start receiver. In order to improve the exploitation efficiency of correlation module in the BeiDou software receiver, a simultaneous multiple BeiDou satellites acquisition algorithm is developed for CDMA signals. The proposed acquisition approach consists of two processes: (i) multiple visible satellites and code phases searching, and (ii) the code phase and carrier frequency identification for individual visible satellites. The block diagram of the proposed acquisition scheme is shown in Fig. 4.

The BeiDou satellites are differentiated by the 37 different PRN codes. The BeiDou B1 ranging code is a subset of the Gold code family, and is generated by means of Modulo-2 addition of G1 and G2 sequences which are respectively derived from 11-bit maximal length linear feedback shift registers, with tap position specified by the generator polynomials:

$$
\begin{aligned}
& G 1(X)=1+X+X^{7}+X^{8}+X^{9}+X^{10}+X^{11} \\
& G 2(X)=1+X+X^{2}+X^{3}+X^{4}+X^{5}+X^{8}+X^{9}+X^{11}
\end{aligned}
$$

The initial phases of G1 and G2 are the same, which are shown as follows:

\section{G1: 01010101010 \\ G2: 01010101010}

Each satellite has a unique ranging code with a different phase shift of G2 sequence, which is generated by respective tapping in the shift register of G2 sequence. The ranging code generation for each satellite can be expressed as follows:

$G_{P R N}=G 1 \oplus\left(G 2\left(s_{p r n 1}\right) \oplus G 2\left(s_{p r n 2}\right)\right)$

where $G_{P R N}$ is Gold code sequence of the prnth BeiDou satellite. $s_{p r n 1}$ and $s_{p r n 2}$ are signal tap values for phase assignment of G2 sequence predefined for prnth satellite.

With a BeiDou PRN sequences generator, all 37 different PRN sequence for BeiDou can be generated. The generation and saving of all possible PRN sequences are aimed to directly load and produce replica code offline instead of generating every time in proposed acquisition algorithm. The first step toward simultaneous multiple phases acquisition is to generate a combined BeiDou replica codes, which is one of the key elements in the proposed acquisition algorithm.

For the purpose of realizing a simultaneous multiple code phases acquisition function, one cycle of combined code sequences for BeiDou In-phase (I) component have been designed as follows:

$C_{\text {Icom }}=\sum_{j=1}^{N} C_{\text {BeiDou_I }}^{j}\left(s v_{B}\right)$

where $N$ is the combination number for BeiDou satellites. $s v_{B}$ denotes the combined satellite prn of BeiDou. As the above equation shows, the combined code sequence has also been generated with multiple code phases of BeiDou satellites. The combination number determines the simultaneous searching sums of satellites. In general, $N$ can be set to 3-4 (i.e. three to four BeiDou satellite is combined) when the receiver is placed outside where the quality of acquired signal is good (normal $C / N_{0}$ ). Otherwise, the $N$ can be changed to be 1-2 (i.e. one to two BeiDou satellites) when the received signal has a low $C / N_{0}$. Besides, in order to increase the reliability of acquisition algorithm, longer accumulation is needed for the noise increase. The accumulation approach will be explained at the end of this section.

As the combined code sequences have been generated, the procedure of the proposed acquisition approach will be explained.

Firstly, the input signals are mixed with the replica carrier signals of In-phase and Quadrature components respectively, and the mixed result can be expressed as:

$y(n)=I(n)+j \cdot Q(n)$

Secondly, in order to improve efficient of correlation operation, let $y(n)$ utilizes the Fourier transform to implement a transformation from the time domain into the frequency domain. An efficient tool for that is the Fast Fourier Transform (FFT) in a software receiver. The transformed result can be written as:

$Y(k)=\sum_{n=0}^{M-1} y(n) e^{-j 2 \pi k n / M}$

Thirdly, since the mixed signal does not have any frequency component, only the offset or phase of the ranging code needs to be found. In order to search the phases of multiple ranging codes simultaneously, the mixed signal $y(n)$ is correlated with the combined code sequence $C_{\text {Icom }}$. Similarly, the correlation operation in the time domain can be transformed to multiplication calculation of $Y(k)$ and the complex conjugate of $C_{\text {Icom }}$ in the frequency domain, so the correlation results are: 


$$
\begin{aligned}
Z_{I}(k) & =\sum_{n=0}^{M-1 M-1} \sum_{m=0} C_{\text {Icom }}(m) y(m+n) e^{-j 2 \pi k n / M} \\
& =\sum_{m=0}^{M-1} C_{\text {Icom }}(m) e^{j 2 \pi k m / M} \sum_{n=0}^{M-1} y(m+n) e^{-j 2 \pi k(m+n) / M} \\
& =C_{\text {Icom }}^{*}(k) Y(k)
\end{aligned}
$$

Finally, when the correlation representation in the frequency domain is computed, the result in time domain can be derived through Inverse Fast Fourier Transform (IFFT), which can be expressed as:

$Z_{I}(n)=i f f t\left(Z_{I}(k)\right)=\frac{1}{M} \sum_{k=0}^{M-1} e^{j 2 \pi k n / M} \cdot Z_{I}(k)=I_{i}(n)+j \cdot Q_{i}(n)$

where $M$ is the length of $y(n)$. The outputs need to compute the absolute value with the two components since the outputs from an IFFT are complex. The absolute values of the IFFT outputs represent the correlation between the input code and the replica combined code sequences. The correlation results of I component are then integrated over a period of samples. If multiple peaks are present in the correlation, the number of peaks is corresponding to the visible satellite number, and the indexes of multiple peaks mark the found code phases of the combined visible satellites. When the combined satellite signal is absent, the correlation results are made of noises only and none peak will appear. From the procedure of the simultaneous multiple code phases acquisition algorithm, the FFT of correlated signal after multiplying the mixed signal need to be performed only once for multiple combined satellites. Obviously, the more satellites under combination, the more signals can be searched simultaneously for the visible satellites acquisition.

In order to increase the reliability of acquisition algorithm, the correlation results from several periods can be added together to obtain the final results, which will at the expense of a longer acquisition time undoubtedly. Specifically, the sums of the correlation results over several code periods are:

$Z_{I \_ \text {sum }}(k)=Z_{I, 1}(k)+Z_{I, 2}(k)+\cdots+Z_{I, L}(k)$

where $L$ is the integration length, which means the summation code periods.

\section{The phase and frequency identification for individual visible satellite}

The simultaneous multiple BeiDou satellites acquisition algorithm has the capability to detect the presence of multiple visible satellites and their code phases with the combined BeiDou code. However, the code phase value for each individual satellite is needed to determine for replica signals initial generation in tracking loops. In order to solve the problem, the individual phase identification approach for each visible satellite is further presented based on multiple satellites acquisition algorithm. The flow chart of phase identification approach for individual visible satellite is shown in Fig. 5. A search within the PRNs used for the generation of local combined code is performed. It is possible that no satellite is found with a combined code. In this case, all the PRNs forming the replica combined code can be excluded in the further searches. Hence the total time needed for acquisition all PRNs can be reduced.

The steps of the phase and frequency identification for individual visible satellite are summarized as follows:

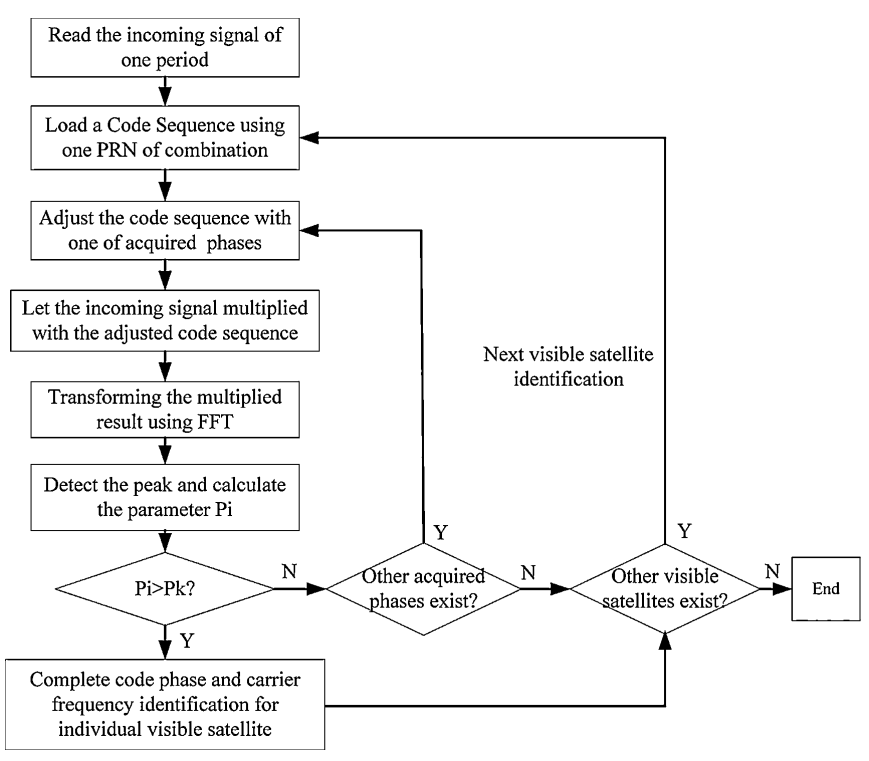

Fig. 5. The phase and frequency identification approach for individual visible satellite.

(1) Read one code period length of the incoming signal. Choose one satellite's PRN from the combination PRNs and load the satellite's code sequence saved locally.

(2) Adjust the phase of the loaded code sequence using the first detected phase derived by the combined acquisition results, so the local code replicas is matched with the corresponding incoming code.

(3) Let the incoming signal got from the step 1 multiplied with the adjusted code sequence, and the next step is transforming the multiplied result through FFT method.

(4) Compute the correlation results and detect the peak value, if the correlation peak value exceeds the predefined threshold, it can be judged that the chosen satellite is visible.

(5) The horizontal axis of the correlation results represents the frequency range, and the peak location can be used to estimate the received signal's carrier frequency of the chosen satellite.

(6) If there exist multiple visible satellites and the used phase is not corresponding to the chosen satellite, so it is necessary to test the other phases and the other combined satellites, which process is shown in Fig. 5.

After the identification of the first visible satellite, the other combined satellites need to be searched till all visible satellites are identified successfully.

The performance of the proposed method in terms of time needed is reduced in two ways: the reduction of search space and the calculation times comparing to other methods. In terms of computation time, it mainly depends on the following parameters:

$N_{I}$ : The number of samples in an integration period such as $L$ (ms), it equals to $L^{*} 16,368$ when the sampling frequency is $16.367667 \mathrm{MHz}$;

$N_{F}$ : The number of frequency searching bins, it equals to 21 when the search space range is $-5 \mathrm{kHz}$ to $5 \mathrm{kHz}$ and the step is $500 \mathrm{~Hz}$; $N_{C}$ : The code phase search time for one code chip, it equals to 2 when the phase resolution is 0.5 chips;

$L_{C}$ : The PRN code length in one code period, it is 2046 chips for BeiDou code sequences.

Given these parameters, the computational loads of the various acquisition methods for N GPS and M BeiDou satellites are compared in Table 1 . The methods implemented in the software 


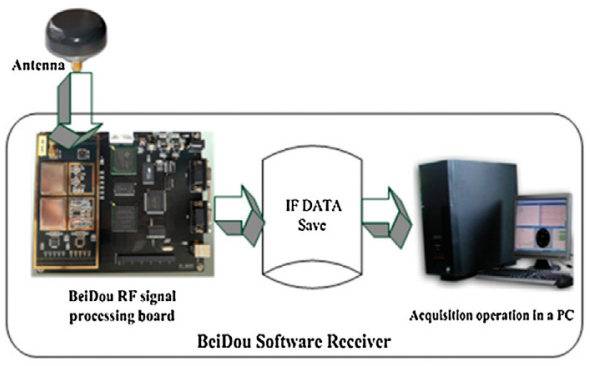

(a) Test setup

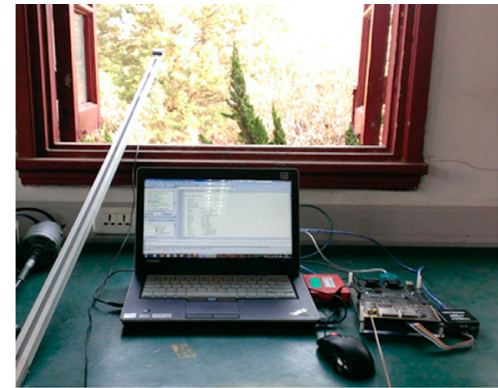

(b) Test scene

Fig. 6. Test setup and scene.

Table 1

The computational complexity comparison of algorithms.

\begin{tabular}{lll}
\hline Algorithms & Multiplications & Sums \\
\hline SSA for BeiDou & $2 N N_{F} L_{C} N_{C} N_{I}$ & $N N_{F} L_{C} N_{C} N_{I}$ \\
PFA for BeiDou & $N\left(L_{C} N_{C} N_{I}+L_{C} N_{C} M_{F F T}\right)$ & $N\left(L_{C} N_{C} A_{F F T}\right)$ \\
PAC for BeiDou & $N N_{F}\left(3 N_{I}+2 M_{F F T}\right)$ & $2 N N_{F} A_{F F T}$ \\
Proposed algorithm & $N_{F}\left(3 N_{I}+2 M_{F F T}\right)+M_{S}$ & $2 N_{F} A_{F F T}+A_{S}$ \\
\hline
\end{tabular}

Table 2

The computational complexity numerical comparison of algorithms.

\begin{tabular}{llll}
\hline Algorithms & Multiplications & Sums & Complexity \\
\hline SSA for BeiDou & $1.125 \times 10^{10}$ & $5.626 \times 10^{9}$ & High \\
PFA for BeiDou & $2.143 \times 10^{9}$ & $3.750 \times 10^{9}$ & Medium \\
PAC for BeiDou & $2.337 \times 10^{7}$ & $3.849 \times 10^{7}$ & Low \\
Proposed algorithm & & & \\
$\quad$ max & $6.366 \times 10^{6}$ & $1.053 \times 10^{7}$ & Low \\
min & $5.973 \times 10^{6}$ & $9.852 \times 10^{6}$ & \\
\hline
\end{tabular}

platform include Serial Search Acquisition (SSA), parallel frequency search acquisition (PFA), parallel code phase search acquisition (PCA) and the proposal method. The parallel frequency search acquisition parallelizes the search for the carrier frequency parameter, which utilizes the Fourier transform to implement a transformation from the time domain into the frequency domain, and only search all the bins in code phase space. If the acquisition could be parallelized in the code phase dimension, this algorithm is simply referred to as parallel code phase search acquisition, which only need to search all the bins in carrier frequency space.

In Table $1, M_{F F T}$ and $A_{F F T}$ are respectively the multiplications and addition computation of FFT according to Cooley-Tukey algorithm. $M_{S}$ and $A_{S}$ are the multiplications and addition computation respectively in the proposed visible satellite's phase and frequency identification method, which maximum and minimum value can be computed as follows:

$$
\left\{\begin{array}{l}
M_{S \max }=N\left(N_{I}+M_{F F T}\right) \\
M_{S \min }=N_{B v}\left(N_{I}+M_{F F T}\right) \\
A_{S \max }=N A_{F F T} \\
A_{\text {Smin }}=N_{B v} A_{F F T}
\end{array}\right.
$$

where $N_{B v}$ is the number of visible BeiDou satellites that are combined to search simultaneously. These four values in Eq. (11) are corresponding to the maximum and minimum multiplications and addition computation when all visible satellites in the combination list are identified successfully. Both the best and worst scenarios are considered. The best scenario is each acquired phase can be matched with the corresponding satellite's PRN from the combination PRNs in the first attempt. The worst scenario is that each

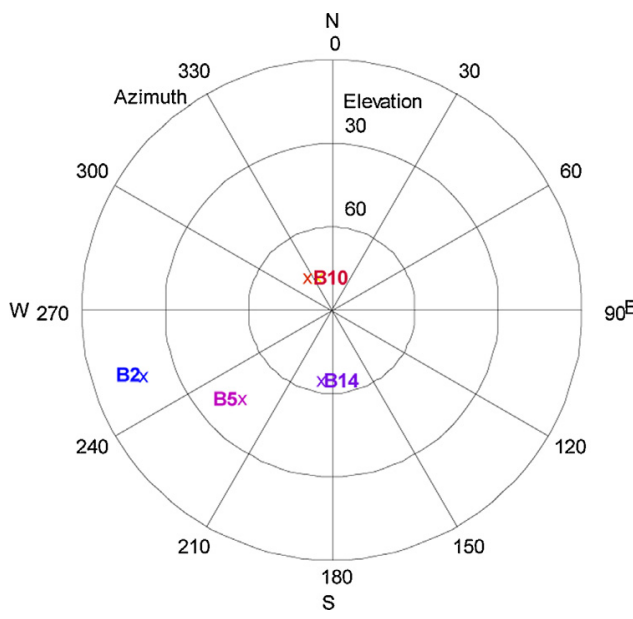

Fig. 7. The skyplot of searched BeiDou satellites.

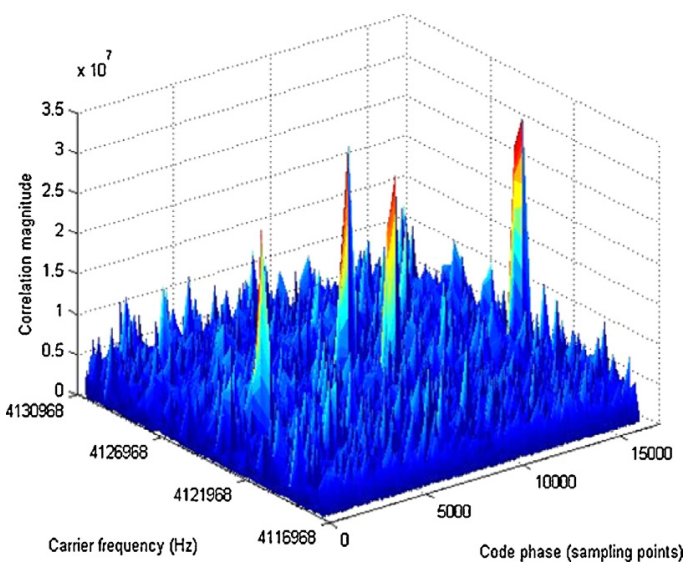

Fig. 8. Multiple BeiDou satellites acquired by the proposed acquisition algorithm.

acquired phase can be successfully matched with the corresponding satellite's PRN in the last attempt of the combined PRN search.

In order to further analyze the computational complexity of different acquisition methods in Table 1 , we provide a numerical example of the computational complexity shown in Table 2. Here, we suppose that the combination number $N=4$, and the number of visible BeiDou satellites $N_{B v}=1$.

Considering the probability of the visible satellites in the combination of BeiDou PRNs, both the best and worst scenarios are calculated. The best scenario is where the combination of nonvisible satellites has higher probability; therefore, minimum time is needed due to the high efficiency in exclusion of non-visible satellites. On the other, the worst scenario needs maximum time. 


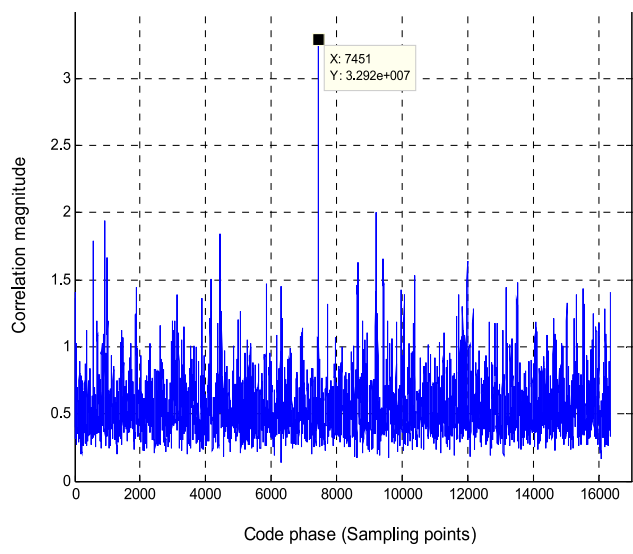

(a) $\mathrm{N}=1$ and $\mathrm{N}_{\mathrm{BV}}=1$

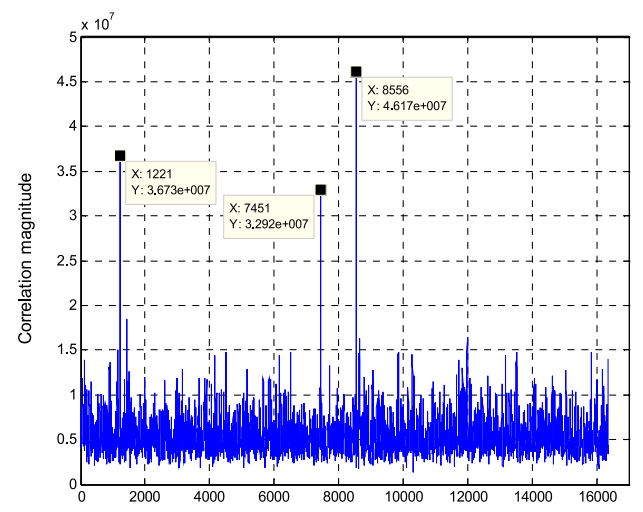

(c) $\mathrm{N}=3$ and $\mathrm{N}_{\mathrm{BV}}=3$

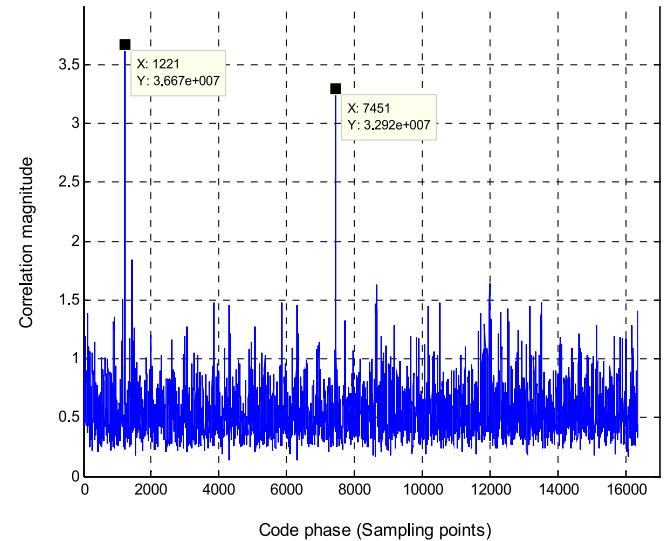

(b) $\mathrm{N}=2$ and $\mathrm{N}_{\mathrm{BV}}=2$

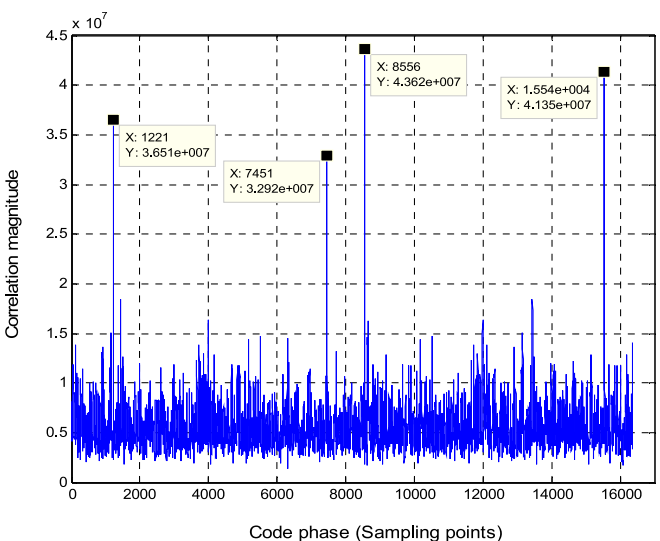

(d) $\mathrm{N}=4$ and $\mathrm{N}_{\mathrm{BV}}=4$

Fig. 9. Multiple code phases acquisition results with different combination numbers.

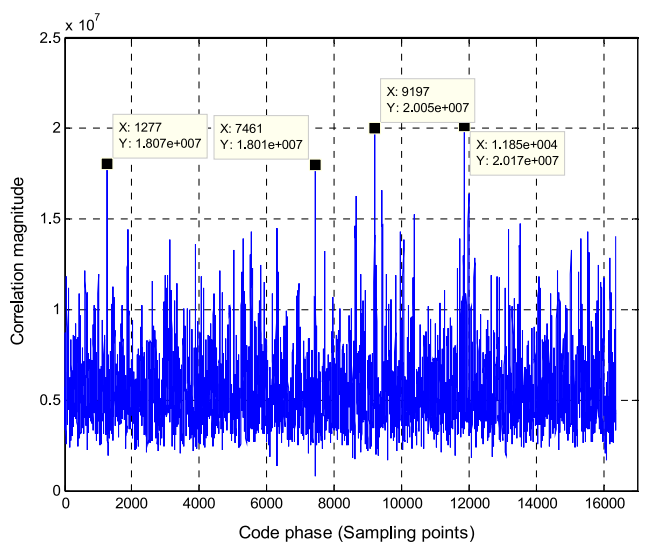

(a) The coherent integration time is $1 \mathrm{~ms}$

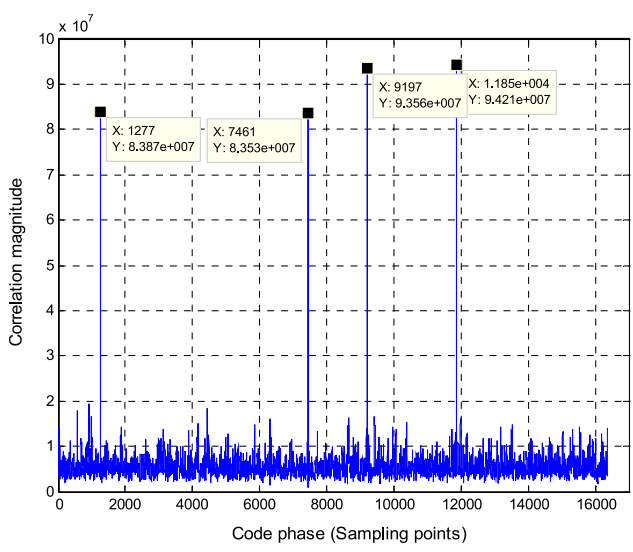

(b) The coherent integration time is $10 \mathrm{~ms}$

Fig. 10. Multiple code phases acquisition results with weak signal.

Table 2 shows that both the PCA method and the proposed acquisition algorithm need less multiplications and additions computation than SSA and PFA methods, and the proposed acquisition algorithm can save about $72.76 \%$ and $72.64 \%$ for multiplications and additions computation load respectively, compared with the PCA method.

\section{Test and results}

In order to evaluate the capability and performance of the proposed acquisition algorithm, two tests were carried out with a high
$C / N_{0}(45 \mathrm{~dB}-\mathrm{Hz})$ signal and a low $C / N_{0}(30 \mathrm{~dB}-\mathrm{Hz})$ signal separately. The test setup is shown in Fig. 6(a). The hardware parts of platform consist of a BeiDou base band signal processing development board including the front end. The output of this board can be saved as a pair of IF data streaming. These data are therefore fed to the BeiDou software receiver for acquisition debugging and algorithms verification in a host computer. The received signals are downconverted to $4.123968 \mathrm{MHz}$ before they are transformed to digital signals by an ADC. Then the corresponding IF signals are samples at $16.367667 \mathrm{MHz}$ in an 8 bits A/D converter. 


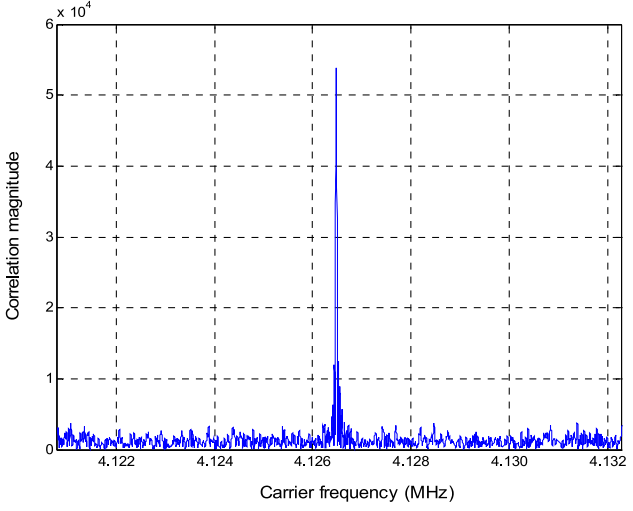

(a) The carrier frequency detection result

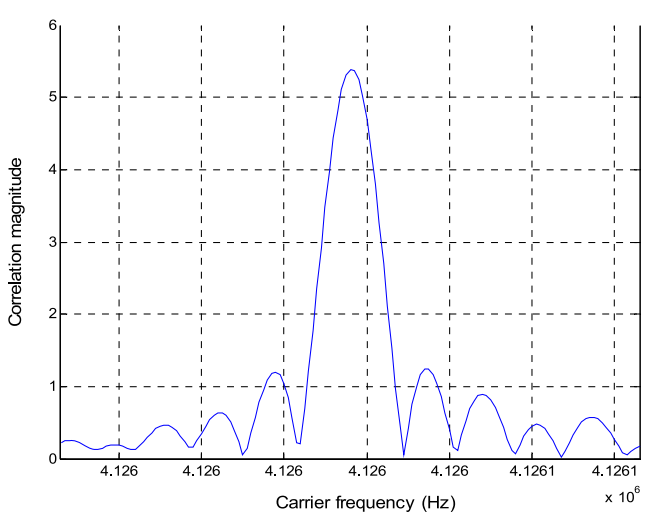

(b) The partial enlargement view

Fig. 11. The carrier frequency detection result and its partial enlargement view.

\subsection{Test scenario}

The signals used for the tests are collected at a static point $\left(118^{\circ}\right.$, $32^{\circ}, 50 \mathrm{~m}$ ) in WGS84, which can be seen in Fig. 6(b). The antenna was placed outside the window to collect a high-quality signal, and a weak signal was collected by the same antenna which was moved to the indoor environment. Moreover, the skyplot of searched satellites is shown in Fig. 7. There are 4 BeiDou satellites (2, 5, 10 and $14)$, and the PRNs of which are in accordance with that defined in BeiDou ICD documents.

\subsection{Test results and analysis}

Firstly, the high $C / N_{0}$ signal was used to test the proposed acquisition algorithm. The combination number $N$ is set to be from 1 to 4 in order to verify the validity of the algorithm. Meanwhile, each corresponding combined code sequence is generated with $N$ visible satellites' PRN using Eq. (3). When there are four visible satellites in the combination list, that is, $N=4$ and $N_{B v}=1$, the multiple satellites acquisition result of the proposed acquisition algorithm is shown in Fig. 8.

As illustrated in Fig. 8, when the local combined code is perfectly aligned with each visible satellite's code in the incoming signal, the output of the Fourier transform will show a distinct peak in magnitude. In order to determine if a satellite signal is acquired successfully, the peak ratio is used, which is defined as the quotient of the highest correlation peak and the second highest peak. The higher the ratio is, the more confident that the signal is acquired correctly. The two horizontal axes of peaks are corresponding to the visible satellite's code phase and carrier frequency respectively. Therefore, the proposed algorithm can acquire four visible BeiDou satellites simultaneously. The corresponding code phase space acquisition results are further shown in Fig. 9 as $N$ is changed from one to four. Since the used signal's strength is high, the coherent integration time is chosen as $1 \mathrm{~ms}$.

Secondly, a low $C / N_{0}$ signal was used to compare the multiple satellites acquisition results with the above results. The code phase acquisition result for four visible BeiDou satellites is shown in Fig. 10(a). As can be seen, the correlation peak values of the four visible satellites are unobvious when the coherent integration time is still set to $1 \mathrm{~ms}$, which directly lead a low detection probability. In order to solve the problem, the larger coherent integration time is used with $10 \mathrm{~ms}$, which correlation result can be seen in Fig. 10(b). Meanwhile, the larger coherent integration time will increase the computational time which is necessary for weak signal acquisition. So the high detection probability and the less computational time are irreconcilable.
Table 3

The execution time of acquisition algorithms.

\begin{tabular}{llrrr}
\hline \multirow{2}{*}{ Algorithms } & \multicolumn{4}{l}{ Visible satellite number } \\
\cline { 2 - 5 } & 1 & \multicolumn{1}{l}{2} & \multicolumn{1}{c}{3} \\
\hline SSA for BeiDou & $8.537 \mathrm{~s}$ & $17.339 \mathrm{~s}$ & $25.216 \mathrm{~s}$ & $32.258 \mathrm{~s}$ \\
PFA for BeiDou & $2.367 \mathrm{~s}$ & $4.614 \mathrm{~s}$ & $7.381 \mathrm{~s}$ & $10.013 \mathrm{~s}$ \\
PAC for BeiDou & $0.970 \mathrm{~s}$ & $1.931 \mathrm{~s}$ & $3.126 \mathrm{~s}$ & $4.023 \mathrm{~s}$ \\
Proposed algorithm & $0.971 \mathrm{~s}$ & $1.763 \mathrm{~s}$ & $2.627 \mathrm{~s}$ & $3.398 \mathrm{~s}$ \\
\hline
\end{tabular}

Table 4

The execution time comparison with different combination number.

\begin{tabular}{lllll}
\hline$N_{B V}$ & $N$ & & & \\
\cline { 2 - 5 } & 1 & 2 & 3 & 4 \\
\hline 1 & $0.971 \mathrm{~s}$ & $1.495 \mathrm{~s}$ & $2.391 \mathrm{~s}$ & $3.052 \mathrm{~s}$ \\
2 & $\times$ & $1.763 \mathrm{~s}$ & $2.516 \mathrm{~s}$ & $3.136 \mathrm{~s}$ \\
3 & $\times$ & $\times$ & $2.627 \mathrm{~s}$ & $3.274 \mathrm{~s}$ \\
4 & $\times$ & $\times$ & $\times$ & $3.398 \mathrm{~s}$ \\
\hline
\end{tabular}

Finally, once the individual visible satellite's code phase is found by the phase and frequency identification approach for individual visible satellite, it is easy to detect the carrier frequency by FFT operation with several milliseconds signal. The carrier frequency detection result and its partial enlargement view are shown in Fig. 11.

Fig. 11 shows that the phase and frequency identification approach has the capability to determine the individual visible satellite's phase and frequency. In contrast, both the PFA and the PCA methods need to perform lots of search steps in each satellite's code phase and carrier frequency dimensions, which will spend more time than the proposed acquisition algorithm.

Consequently, a number of signal acquisition methods have been implemented in the software platform including SSA, PFA, PCA to compare with the proposed algorithm. A comparison of execution time of several acquisition methods for multiple satellites is summarized in Table 3.

As illustrated in Table 3, the acquisition time needed by the proposed algorithm is obviously less than the SSA, PFA and PCA methods. Furthermore, a comparison for execution time of proposed acquisition algorithm with different N and NBV is summarized in Table 4.

It can be seen from Table 4, that the proposed algorithm has the most significant reduction in acquisition time when $N=4, N_{B V}=1$. The results in Table 4 show that the proposed simultaneous multiple satellites acquisition algorithm can reduce the computational time by $8.7 \%\left(N=2, N_{B V}=2\right)$ to $24.1 \%\left(N=4, N_{B V}=1\right)$ compared with the PCA method when $N>1$ depending on different combination 
numbers and different numbers of visible satellites in the combination list. The algorithm proposed can be extended to include GPS signals.

\section{Conclusions}

The main advantage of the proposed algorithm is that multiple BeiDou satellites' signals can be processed together and multiple visible satellites can be detected simultaneously. Finally, the experimental results show that the proposed acquisition algorithm has an effective performance for BeiDou signals acquisition and can significantly reduce the computational load comparing to the serial search, parallel frequency acquisition and parallel code phase acquisition methods for multiple satellites' signals acquisition.

The nature of the new local code is a combination of multiple CDMA codes. This method can be adopted in a software receiver for a single constellation where the local code is a combination of PRNs from the same constellation. This method can also be extended to multiple constellations using CDMA signals. In this case, the local code can be formed from PRNs of all available constellations.

\section{Acknowledgments}

This work was supported by the National Natural Science Fund of China (Grant No. 61374115 and 61273057), Natural Science Fund of Jiangsu Higher Education Institution (15KJB510016), IndustryAcademy-Research Fund Project supported by AVIC. The author thanks the anonymous reviewers for their comments and valuable remarks.

\section{References}

[1] A. Polydoros, C.L. Weber, A unified approach to serial search spread-spectrum code acquisition - part I and II, IEEE Trans. Commun. 60 (1) (1984) 542-549, http://dx.doi.org/10.1109/TCOM.1984.1096109.

[2] V. Patel, P. Shukla, Faster methods for GPS signal acquisition in frequency domain, in: Proceedings of International Conference on Emerging Trends in Networks and Computer Communications (ETNCC), 2011, pp. 84-88.

[3] P. Huang, Y. Pi, Research on novel structure of GPS signal acquisition based on software receiver, in: Intelligent Signal Proceeding and Communication Systems (ISPACS), 2010, pp. 1-4.

[4] A.J.R.M. Coenen, D.J.R. van Nee, Novel fast GPS/GLONASS code-acquisition technique using low update rate FFT, Electron. Lett. 28 (9) (1992) 863-865.

[5] Y. Li, T. Ma, T. Tian, An acquisition scheme for non-coherent DSSS signal, in: Proceedings of Wireless Communications Networking and Mobile Computing, 2010, pp. 1-4.

[6] D. Borio, C. O'Driscoll, G. Lachapelle, Composite GNSS signal acquisition over multiple code periods, IEEE Trans. Aerospace Electron. Syst. 46 (1) (2010) 193-206.

[7] C. Yang, T. Nguyen, E. Blasch, M. Miller, Post-correlation semi-coherent integration for high-dynamic and weak GPS signal acquisition, in: ION PLANS 2008, San Mateo, CA, 2008, pp. 1341-1349.

[8] H. Elders-Boll, U. Dettmar, Efficient differentially coherent code/Doppler acquisition of weak GPS signals, in: IEEE 8th International Symposium on Spread Spectrum Techniques and Applications, 2004, pp. 731-735.

[9] X. Ba, Y. Yang, J. Li, J. Chen, Differentially coherent acquisition algorithm for indoor GPS, in: Proceedings of IET International Conference on Wireless, Mobile and Multimedia Networks, 2006, pp. 1-4.

[10] S.K. Shanmugam, J. Nielsen, G. Lachapelle, Enhanced differential detection scheme for weak GPS signal acquisition, in: Proceedings of ION GNSS 2007, Forth Worth, 2007, pp. 189-202.

[11] J. Qiu, Y. Qian, R. Zheng, Non-coherent, differentially coherent and quasicoherent integration on GNSS pilot signal acquisition or assisted acquisition, in: ION PLANS 2012, 2012, pp. 989-997.

[12] N. O'Mahony, C.M. Colin, Constrained optimization of coincidence detector parameters for GPS acquisition, IEEE Trans. Commun. 60 (1) (2012) 176-188.

[13] D. Borio, L. Camoriano, L. Lo Presti, Impact of GPS acquisition strategy on decision probabilities, IEEE Trans. Aerospace Electron. Syst. 44 (3) (2008) 996-1011.

[14] C. O'Driscoll, Performance analysis of the parallel acquisition of weak GPS signals (Ph.D. thesis), National University of Ireland, Ireland, 2007.

[15] X. Wang, X. Ji, S. Feng, A scheme for weak GPS signal acquisition aided by SINS information, GPS Solut. (2013), http://dx.doi.org/10.1007/s10291-013-0325-9.

[16] BeiDou Navigation Satellite System Signal in Space Interface Control Document-Open Service Signal B1I (Version 2.0), China Satellite Navigation Office, December 2013, pp. 1-15. 